BULL. AUSTRAL. MATH. SOC.

VOL. $23(1981), 181-190$.

\title{
TREES AS COMMUTATIVE BCK-ALGEBRAS
}

\author{
WILLIAM H. CORNISH
}

\begin{abstract}
A new method of constructing commutative $\mathrm{BCK}$-algebras is given. It depends upon the notion of a valuation of a lower semilattice in a given comutative BCK-algebra. Any tree with the descending chain condition has a valuation in the natural numbers, considered as a commutative $\mathrm{BCK}-a$ lgebra; the valuation is the height-function. Thus, any tree of finite height possesses a uniquely determined commutative BCK-structure. The finite trees with at most one atom and height at most $n$ are precisely the finitely generated subdirectly irreducible (simple) algebras in the subvariety of commutative BCK-algebras which satisfy the identity $\left(\mathrm{E}_{\mathrm{n}}\right): x y^{n}=x y^{n+1}$. Due to congruence-distributivity, it is then possible to describe the associated lattice of subvarieties.
\end{abstract}

\section{Introduction}

The concept of a lower semilattice with a valuation in a commutative BCK-algebra is introduced, and it is shown that such a semilattice can be converted into a commutative BCK-algebra. Any tree, which satisfies the descending chain condition, provides an example; the valuation is the height-function. Thus, any tree of finite height possesses a uniquely determined, commutative-BCK-algebra-structure. It is then possible to

Received 15 September 1980. The author would like to thank Professor Kiyoshi Iséki for his kindness and encouragement, Brian Davey for a preprint and reprint of his paper [6], and Henry Rose for lively discussions on congruence-distributive varieties. 
completely describe the lattice of subvarieties of the variety of commutative BCK-algebras satisfying the identity $x y^{n}=x y^{n+1}$.

\section{Valuations}

Because of Yutani [15], a commutative BCK-algebra can be considered as a groupoid with a nullary operation 0 , which satisfies the identities: $x x=0, x 0=x, x(x y)=y(y x), \quad(x y) z=(x z) y$. We will presume a familiarity with BCK-algebras and especially commutative BCK-algebras; good references are supplied by Iséki and Tanaka [9] and Traczyk [14], but see also [12], [3], [4] and [5].

Let $(A ; \wedge, 0)$ be a lower semilattice with smallest element 0 , and $(C ; 0)$ be a commutative BCK-algebra. Then, the semilattice $A$ is said to have a valuation, $v$, in the commutative BCK-algebra $C$ if $v$ is a function mapping $A$ into $C$ such that

(v1) $v(a \wedge b)=v(a) \wedge v(b)$ for any $a, b \in A$, which possess $a$ common upper bound;

(v2) for any $a \in A$, the restriction $v_{a}$ of $v$ to the interval $[0, a]$ possesses an inverse

$$
v_{a}^{-1}:[0, v(a)] \rightarrow[0, a] ;
$$

(v3) for any $a, b \in A$, with $a \leq b$, and $x \in[0, v(a)]$ $v_{a}^{-1}(x)=v_{b}^{-1}(x)$.

Any commutative BCK-algebra is a lower semilattice, wherein the infimum is given as the derived operation $x \wedge y=x(x y)=y(y x)$. Thus, (VI)-(V3) make sense. Also, each interval $[0, x]$ in a commutative BCKalgebra is a distributive lattice; cf. [3, Section 3], [14]. Due to (VI) and (v2), $v_{a}$ and $v_{a}^{-1}$ are then mutually inverse lattice-isomorphisms. Also $v$ is isotone and $v(0)=0$.

THEOREM 1.1. Let $(A ; \wedge, 0)$ be a Lower semilattice which possesses a valuation $v$ in a commutative BCK-algebra $(C ; 0)$. Define a binary operation on $A$ by 


$$
a b=v_{a}^{-1}(v(\alpha) v(a \wedge b))
$$

With respect to this operation, $A$ is a commutative BCK-algebra and the original semilattice infimm is given by $a \wedge b=a(a b)=b(b a)$.

Moreover, for each $a \in A, v_{a}$ and $v_{a}^{-1}$ are mutually inverse BCKisomorphisms between the BCK-subalgebras $([0, a] ; 0)$ and $([0, v(a)] ; 0)$.

Proof. We must show that Yutani's identities hold. However, before doing this, we should note that (V1) and (V2) imply that $v(a b)=v(a) v(a \wedge b)$ and $a b \leq a$ for any $a, b \in A$.

As $v_{a}^{-1}(0)=0$,

$$
a a=v_{a}^{-1}(v(a) v(a \wedge a))=v_{a}^{-1}(v(a) v(a))=v_{a}^{-1}(0)=0 .
$$

As $v(0)=0$,

$$
a 0=v_{a}^{-1}(v(a) v(a \wedge 0))=v_{a}^{-1}(v(a) 0)=v_{a}^{-1}(v(a))=a \text {. }
$$

Due to $\left(v_{3}\right), v_{a}^{-1}(v(a \wedge b))=v_{a \wedge b}^{-1}(v(a \wedge b))=a \wedge b$. Hence,

$$
\begin{aligned}
a(a b)= & v_{a}^{-1}(v(a) v(a \wedge(a b)))=v_{a}^{-1}(v(a) v(a b))=v_{a}^{-1}(v(a)(v(a) v(a \wedge b))) \\
& =v_{a}^{-1}(v(a) \wedge v(a \wedge b))=v_{a}^{-1}(v(a \wedge b))=v_{a \wedge b}^{-1}(v(a \wedge b))=a \wedge b .
\end{aligned}
$$

As $a \wedge b=b \wedge a, a(a b)=b(b a)$.

Because $a b \leq a$, (VI) implies that

$$
v((a b) \wedge c)=v((a b) \wedge(a \wedge c))=v(a b) \wedge v(a \wedge c) \text {. }
$$

Hence,

$$
\begin{aligned}
(a b) c=v_{a b}(v(a b) & v((a b) \wedge c))=v_{a b}(v(a b)(v(a b) \wedge v(a \wedge c))) \\
& =v_{a b}(v(a b) v(a \wedge c))=v_{a b}((v(a) v(a \wedge b)) v(a \wedge c)) \\
& =v_{a}((v(a) v(a \wedge b)) v(a \wedge c))=v_{a}((v(a) v(a \wedge c)) v(a \wedge b)) .
\end{aligned}
$$

Because of the symmetric roles of $b$ and $c$, we conclude that $(a b) c=(a c) b \cdot$ Thus $A$ is a commutative BCK-algebra. 
Finally suppose $b, c \in[0, a]$. Due to (V1) and (V3), $b c=v_{b}^{-1}(v(b) v(b \wedge c))=v_{b}^{-1}(v(b)(v(b) \wedge v(c)))$

$$
=v_{b}^{-1}(v(b) v(c))=v_{a}^{-1}\left(v_{a}(b) v_{a}(c)\right) \text {. }
$$

That is, $v_{a}(b c)=v_{a}(b) v_{a}(c)$, and so $v_{a}:[0, a] \rightarrow[0, v(a)]$ is a BCKisomorphism.

When $(A ; 0)$ is a commutative BCK-algebra and $(A ; \wedge, 0)$ is its lower semilattice reduct, the identity function on $A$ provides a valuation of $(A ; \wedge, 0)$ in the BCK-algebre $(A ; 0)$. We now give less trivial examples.

EXAMPLE 1.2. Consider the unit interval $[0,1]$ of the real numbers as a commutative BCK-algebra, wherein $x y=\max (x-y, 0)=x-\min (x, y)$. Let $(A ; \wedge, 0)$ be the tree with two distinct maximal chains $\{a(x): x \in[0,1]\},\{b(x): x \in[0,1]\}$, each of which is orderisomorphic to $[0,1]$, and such that $a(y)=b(y)$, when $y \in\left[0, \frac{z}{2}\right]$, while $a(z) \wedge b(w)=a\left(\frac{1}{2}\right)=b\left(\frac{1}{2}\right)$ for all $z, w \in\left(\frac{1}{2}, 1\right]$. Then $v: A \rightarrow[0,1]$, defined by $v(a(x))=v(b(x))=x$ for all $x \in[0,1]$, is a valuation.

EXAMPLE 1.3. Let $C$ be a commutative BCK-algebra and for each $i$ in an index set $I$ with at least two elements, let $C_{i}$ be a copy of the underlying semilattice of $C$. Form the semilattice $(A ; \wedge, 0)$ where $A=\cup\left\{C_{i}: i \in I\right\}$ and $C_{i} \cap C_{j}=\{0\}$ if $i \neq j$. Each $C_{i}$ is orderisomorphic to $C$ under $v_{i}$, say, and $a$ and $b$ are incomparable when $a \in C_{i}, b \in C_{j}$ and $i \neq j$. Then $v: A \rightarrow C$, given by $v(a)=v_{i}(a)$ if $a \in C_{i}$, is a valuation. When $C$ is taken as the 2-element BCKchain, the resulting BCK-algebra is the one given in Example 3 of Iseki and Tanaka [8]. When $C$ is the BCK-algebra which is the set of natural numbers $N=\{0,1,2, \ldots\}$ with $B C K$-product $a b=\max (a-b, 0)$, the resulting $\mathrm{BCK}-a l g e b r a$ is the one constructed in Example 4 of Iséki and Tanaka $[8]$.

By a tree, we mean a lower semilattice $(A ; \wedge, 0)$ with a smallest element 0 , in which any two elements have a common upper bound only if 
they are comparable or equivalently, each initial interval $[0, a]$ is a chain. When a tree $(A ; \wedge, 0)$ satisfies the descending chain condition, each element $a \in A$ has finite height $h(a) ; h(a)$ is the length of the chain $[0, a]$. A tree has finite height equal to $n$, if $n$ is the maximum of the lengths of its subchains.

Let $(N ; 0)$ be the commutative BCK-algebra, wherein $N=\{0,1,2, \ldots\}$ is the set of natural numbers and the BCK-product on $N$ is given by $x y=\max (x-y, 0)=x-\min (x, y)$, for each $x, y \in N$. We are now ready to give the most important instance of Theorem 1.1; we formulate it as a theorem.

THEOREM 1.4. Let $(A ; \wedge, 0)$ be a tree with the descending chain condition and let $v: A \rightarrow N$ be given by $v(a)=h(a)$ for each $a \in A$. Then $v$ is a valuation of the tree $(A ; \wedge, 0)$ in the commutative BCKalgebra $(N ; 0)$. Thus the tree $A$ becomes a commitative BCK-algebra, wherein the $\mathrm{BCK}$-product $a b$ of $a, b \in A$ is the unique element of height $h(a)-h(a \wedge b)$ in the interval $[0, a]$. What is more, this is the only product which is definable on $A$ so that the resulting structure is a commutative BCK-algebra, whose lower semilattice reduct coincides with the original semilattice $(A ; \wedge, 0)$.

Proof. We only have to establish the uniqueness of the BCK-structure. Suppose $(A ; *, 0)$ is a commutative BCK-algebra such that the original infimum is given by $a \wedge b=a^{*}\left(a^{*} b\right)=b^{*}\left(b^{*} a\right)$, for any $a, b \in A$. Then the finite chain $[0, a]$ is a subalgebra of $\left(A ;{ }^{*}, 0\right)$ and $a, a \wedge b \in[0, a]$. But Traczyk [14, Theorem 3.5] has shown that there is a unique way to turn a finite chain into a commutative BCK-algebra so that the original order and the induced BCK-order coincide. Hence $a^{*}(a \wedge b)=a(a \wedge b)$. But in $(A ; *, 0), a^{*}(a \wedge b)=a^{*} b$ and, in $(A ; 0), a(a \wedge b)=a b$. Hence $a^{*} b$ is the unique element of height $h(a)-h(a \wedge b)$ in $[0, a]$, as asserted.

Some examples of trees of finite height supporting a commutative BCKstructure have already been studied; see, for example, Iséki and Tanaka [8, Example 5] and Setó [13].

We now exploit Theorem 1.4 to study the lattice of subvarieties of a certain variety of commutative BCK-algebras. 


\section{Lattice of subvarieties}

For $n \geq 0$, the polynomials $x y^{n}$ are defined inductively by $x y^{0}=x, x y^{k+1}=\left(x y^{k}\right) y$.

LEMMA 2.1. Let $(A ; 0)$ be a commutative BCK-algebra whose underlying semilattice is a tree with the descending chain condition. Let $a, b \in A$ and $n$ be a natural number. Then

$$
h\left(a b^{n}\right)=\max (h(a)-n h(a \wedge b), 0) .
$$

Moreover, if $a \wedge b>0$ then $a b^{h(a)}=0$ and $h(a) \geq 1$.

Proof. The second assertion is an immediate consequence of the first assertion. We use induction to establish the first one.

It is evidently true for $n=0$. Suppose $m \geq 0$ and

$$
h\left(a b^{m}\right)=\max (h(a)-m h(a \wedge b), 0)
$$

Then

$h\left(a b^{m+1}\right)=h\left(\left(a b^{m}\right) b\right)=h\left(a b^{m}\right)-h\left(\left(a b^{m}\right) \wedge b\right)=h\left(a b^{m}\right)-h\left(\left(a b^{m}\right) \wedge(a \wedge b)\right)$

$=h\left(a b^{m}\right)-\min \left(h\left(a b^{m}\right), h(a \wedge b)\right)=\max \left(h\left(a b^{m}\right)-h(a \wedge b), 0\right)$

$=\max (\max (h(a)-\operatorname{mh}(a \wedge b), 0)-h(a \wedge b), 0)$

$=\max (\max (h(a)-(m+1) h(a \wedge b),-h(a \wedge b)), 0)=\max (h(a)-(m+1) h(a \wedge b), 0)$.

The proof is now complete.

We now come to the important role played by trees.

THEOREM 2.2. Let $(A ; \wedge, 0)$ be a lower semilattice with smallest element 0 , which satisfies the descending chain condition. Then the following conditions are equivalent:

(i) A is a reduct of a subdirectly irreducible commutative BCK-algebra;

(ii) $A$ is a reduct of a simple commutative BCK-algebra;

(iii) $A$ is a tree in which 0 is meet-irreducible.

Proof. Because of Theorem 1.4 and Lemma 2.1, (iii) implies (i), in view of the correspondence between ideals and congruences in any variety of BCK-algebras. For this correspondence, see the remarks of [4] which 
immediately precede Theorem 2.4, therein; the observation on simplicity is an immediate consequence, $c f$. the proof of [4, Corollary 3.2] and also Iséki [7, Proposition 4].

of course, (ii) follows from (i). The implication $(i) \Rightarrow$ (iii) is the content of Lemmas 5.1 and 5.2 of Romanowska and Traczyk [12]. The fact that (i) implies that 0 is meet-irreducible is their Lemm 5.1; for a different explanation involving the notion of prime ideal, see [5, Theorem 4.3]. Why does ( $i$ ) then imply that $A$ is a tree? Well, for each $a \in A$, $[0, a]$ is a lattice with the map $b \rightarrow a b(b \in[0, a])$ as an involution, due to the commutativity of $A$, and so $a$ is then join-irreducible in $[0, a]$. Thus $[0, a]$ is a chain, and the underlying semilattice is a tree. This argument is due to Romanowska and Traczyk [12, Lemma 5.2].

COROLLARY 2.3. A commutative BCK-algebra of finite height is subdirectly irreducible (simple) if and only if it is a tree with a unique atom, endowed with the BCK-structure of Theorem 1.4 .

In [4], the author showed that the class of BCK-algebras, satisfying the identity $\left(E_{n}\right): x y^{n}=x y^{n+1}$, is a congruence-distributive variety.

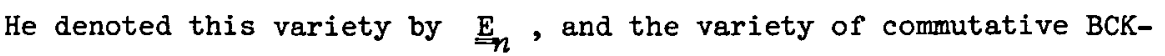
algebras by $\underline{\underline{T}}$. The variety $\underline{\underline{T}}$ is also congruence-distributive, see [3, Section 3] for a list of proofs; in [4, Theorem 3.3], the author extended the proof of [4, Theorem 2.1] to show that any quasicommutative variety of $B C K-a l g e b r a s$ is, in fact, congruence-3-distributive. In order to conform with the notation of [4] and [5], the variety of commutative BCK-algebras, satisfying the identity $\left(\mathrm{E}_{\mathrm{n}}\right)$ is denoted by $\underline{\underline{\mathrm{T}}} \cap \underline{\underline{E}_{n}}$. The fundamental result on the subdirectly irreducible algebras in this variety has been proved by Komori [10, Theorem 3.13] and is discussed immediately before Lemma 3.4 in [4]. Using Theorems 1.4, 2.1, and Corollary 2.3, together with Theorem 2.4, we can state:

THEOREM 2.4. The subdirectly irreducible (simple) algebras in the variety $\stackrel{\mathrm{T}}{\mathrm{I}} \underset{\mathrm{F}}{\mathrm{E}}$ are precisely those trees of height less than or equal to $n$, which possess a unique atom and whose BCK-stmucture is determined by Theorem 1.4 .

Proof. Komori's [10, Theorem 3.13] says that a commutative BCK-chain, 
which satisfies $\left(\mathrm{E}_{\mathrm{n}}\right)$, must have at most $n$ elements.

Because of the isomorphism in Theorem 1.1, it is not hard to see that the set of maximal elements and the unique atom form a generating set of a subdirectly irreducible algebra having finite height. Sometimes, the unique atom can be omitted, but no maximal element can ever be eliminated.

Hence, we obtain:

THEOREM 2.5. Each finitely generated subdirectly irreducible algebra in the variety $\mathrm{T} \cap \underline{\mathrm{E}}_{n}$ is both simple and finite. Consequently, the variety $\underline{\underline{T}} \cap \underline{\underline{E}}$ is locally finite, that is, each of its finitely generated subalgebras is finite.

Proof. There are only finitely many finite trees of height $n$.

The number of non-isomorphic finite trees having a given number of elements was determined by Cayley in 1857, according to Knuth [11, p. 405]. By adding a new smallest element to a finite tree, we produce a finite subdirectly irreducible algebra. Hence, Cayley's work applies to the

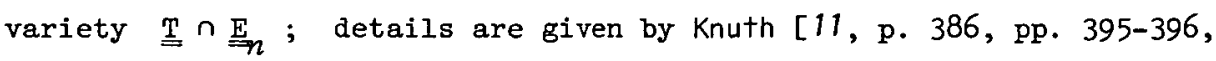
Exercises 1-4].

As we mentioned after Corollary 2.3, the variety $\underline{\underline{\underline{T}}} \cap \underset{\underline{E_{n}}}{\text { is }}$ congruence-distributive. This fact and Theorem 2.5 allow us to apply Theorem 3.3 of Davey [6]: the lattice of subvarieties of a locally finite congruence-distributive variety is isomorphic to the lattice of all hereditary subsets of the partially ordered set of isomorphism-classes of the finite subdirectly irreducible algebras; for two representative such algebras $A$ and $B, A \leq B$ if and only if $A$ is a homomorphic image of a subalgebra of $B$. Combining this with Theorem 2.4, we obtain:

THEOREM 2.6. Let $P_{n}$ be the partially ordered set of isomorphismclasses of finite trees with a unique atom and height at most $n$; for two representative such trees $A$ and $B, A \leq B$ if and only if $A$ is isomorphic to a subtree of $B$ under a semilattice-homomorphism which preserves smallest elements. Then the lattice of subvarieties of the variety $\stackrel{\underline{T}}{\underline{\underline{E}}}$ is isomorphic to the lattice of hereditary subsets of $P_{n}$ 
Moreover, each algebra in $\underset{\underline{T}}{\underline{\underline{E}}} \underline{\underline{h}}$ is isomorphic to a subalgebra of a direct power of the tree of height $n$ having at most one atom and countable many elements covering each of its elements of height $1, \ldots, n-1$, if $n \geq 2$, endowed with the BCK-stmucture of Theorem 1.4.

The variety $\underline{\underline{\underline{T}}} \cap \underline{\underline{E}}_{1}$ is the variety of implicative BCK-algebras. Theorem 2.6 gives the well known result that this variety is equationally complete and generated by the 2-element algebra. For a history see [2]; another proof was given recently by Comer [1].

From Theorem 2.6 it also follows that the lattice of subvarieties of $\underline{\underline{T}} \cap \underline{\underline{E}}_{2}$ is a chain of type $\omega+I$. This was established by the author in [5, Theorem 5.4], using a different approach. In [5, Theorem 5.3], an equational base was given for each subvariety of $\underline{\underline{\underline{T}}} \cap \underline{\underline{E_{2}}}$ : the variety generated by the tree of height 2 with one atom and $n \geq 1$ maximal elements has an equaltional base which consists of a base for $\stackrel{T}{=} \underline{\underline{E}}_{2}$ together with the identity

$$
\left(s_{n}\right): \bigwedge_{1 \leq i \leq n}\left(x_{i} x_{i+1}\right) \wedge x_{n+1} x_{1}=0 .
$$

It would be interesting to find an equaltional base for the variety generated by a finite (simple) tree.

\section{References}

[1] Stephen D. Comer, "The representation of implicative BCK-algebras", Math. Japonica 25 (1980), 111-115.

[2] William H. Cornish, "A multiplier approach to implicative BCKalgebras", Math. Sem. Notes Kobe Univ. 8 (1980), 157-169.

[3] William H. Cornish, "3-permutability and quasicommutative BCKalgebras", Math. Japonica 25 (1980), 477-496.

[4] William H. Cornish, "Varieties generated by finite BCK-algebras", Bull. Austral. Math. Soc. 22 (1980), 411-430.

[5] William H. Cornish, "On Iséki's BCK-algebras", Proceedings of the Western Australian Algebra Conference, Perth, 1980 (to appear). 
[6] Brian A. Davey, "On the lattice of subvarieties", Houston J. Math. 5 (1979), 183-192.

[7] Kiyoshi Iséki, "On finite BCK-algebras", Math. Japonica 25 (1980), 225-229.

[8] Kiyoshi Iséki and Shotaro Tanaka, "Ideal theory of BCK-algebras", Math. Japonica 21 (1976), 351-366.

[9] Kiyoshi Iséki and Shotaro Tanaka, "An introduction to the theory of BCK-algebras", Math. Japonica 23 (1978), 1-26.

[10] Yuichi Komori, "Super-Zukasiewicz implicational logics", Nagoya Math. J. 72 (1978), 127-133.

[11] Donald E. Knuth, The art of computer programing. Volume 1: Fundamental algorithms, 2nd edition (Addison-Wesley, Reading, Massachusetts; Menlo Park, California; London; 1973).

[12] Anna Romanowska and Tadeusz Traczyk, "On commutative BCK-algebras", preprint.

[13] Yasuo Setō, "Some examples of BCK-algebras", Math. Sem. Notes Kobe Univ. 5 (1977), 397-400.

[14] Tadeusz Traczyk, "On the variety of bounded commutative BCK-algebras algebras", Math. Japonica 24 (1979), 283-292.

[15] Hiroshi Yutani, "On a system of axioms of a commutative BCK-algebra", Math. Sem. Notes Kobe Univ. 5 (1977), 255-256.

School of Mathematical Sciences,

FIinders University of South Australia, Bedford Park,

South Australia 5042,

Australia. 